\title{
A Randomized Controlled Trial Testing the Effects of Singapore's Front-of-Pack Healthier Choice Symbol Label With or Without a Physical Activity Equivalent Label on Food Purchases and Measures of Diet Quality
}

Eric Finkelstein ( $\square$ eric.finkelstein@duke-nus.edu.sg )

Duke-NUS Graduate Medical School https://orcid.org/0000-0001-6443-9686

Brett Doble

Duke-NUS Medical School

Felicia Jia Ler Ang

Duke-NUS Medical School

Wei Han Melvin Wong

Duke-NUS Medical School

Rob M. van Dam

National University Singapore Saw Swee Hock School of Public Health

Research

Keywords: Front-of-pack label, Nutrition label, Physical Activity Equivalent, Food Intake, Calories, Online Grocery Store, Healthier Choice, Diet

Posted Date: December 16th, 2019

DOl: https://doi.org/10.21203/rs.2.18782/v1

License: (9) This work is licensed under a Creative Commons Attribution 4.0 International License.

Read Full License 


\section{Abstract}

\section{Background}

Singapore's front-of-pack (FOP) Healthier Choice Symbol (HCS) label is an easy to understand signal to consumers of how they can make a healthier choice within a given food category. We assess its effectiveness at influencing food purchases and diet quality.

\section{Methods}

Randomized controlled trial using a $3 \times 3$ within-subject crossover design with adult Singapore residents recruited online. Each participant shopped once in three conditions on an experimental online grocery store in random order: 1) no FOP control; 2) Similar to Arm 1 except select products displayed HCSs, as would occur in stores in Singapore; 3) Similar to Arm 2 with additional information displaying Physical Activity Equivalents (PAEs) per serving of each product. Participants with minimum of one control and one intervention condition shop were analyzed. First-differenced regressions on calories per serving (primary) and other measures of diet quality were used to compare purchasing behavior across conditions.

Results

From January 2019 to April 2019, 117 participants were randomized: 10 (8.5\%) completed one shop; 2 completed two shops (1.7\%); and 105 (89.7\%) completed all three, resulting in 317 unique shops. The HCS, without PAEs, led to a statistically significant five-percentage point increase in the proportion of HCS products purchased. However, we could not reject the null hypothesis of no difference in calories per serving in either HCS $(95 \% \mathrm{Cl},-10 \cdot 63: 20 \cdot 01)$ or when combined with PAEs $(95 \% \mathrm{Cl},-5 \cdot 25: 21 \cdot 54)$ or differences in any of the diet quality measures assessed.

\section{Conclusions}

The HCS influences purchasing patterns, but does not, either alone or in combination with a PAE label, appear to reduce caloric intake or improve overall diet quality. These findings suggest that the HCS label, as currently applied, may be the wrong label for addressing rising rates of obesity and non-communicable diseases in Singapore.

\section{Background}

Like many affluent nations, Singapore has experienced steadily increasing rates of obesity, diabetes and other diet-related chronic health conditions, including cardiovascular disease, cancer, and renal failure (1). As a result, Singapore has adopted a number of different interventions aimed at encouraging healthier food and beverage consumption. One such strategy is front-of-package (FOP) nutrition labelling. 
Since 2001, Singapore has supplemented the Nutrition Information Panel (NIP) appearing on many products with a simple FOP Healthier Choice Symbol (HCS), which serves as a signal to consumers as to which products are healthier options within a specific category (e.g., which are the healthier biscuits or the healthier beverages). Manufacturers must meet category-specific criteria before a product can display the HCS and an associated tagline. There are up to 30 different taglines to choose from, each focusing on a different dimension of diet quality (Fig. 1).

The rationale behind the HCS is that it provides an easy to understand signal to consumers of how they can make a healthier choice within a given food or beverage category. However, the HCS has some potentially negative consequences, especially when it comes to caloric intake, the primary contributor to obesity and diabetes. First, because the HCS offers a signal that a product is 'healthier' (2), it is possible that people will overconsume HCS products. It is even possible that people will consume more products without a HCS as a result of also purchasing more products with a HCS as consumers might think they are entitled to some less healthy products as a result of making 'healthier' choices for other products.

Moreover, only a handful of the taglines accompanying the HCS directly relate to calories. If consumers considered HCS with these taglines only and engaged in no offsetting behavior then they would consume fewer calories. However, if consumers positively respond to all HCSs and associated taglines, then their net caloric intake may be no lower, and perhaps even higher, than had they ignored the HCSs altogether. This results because some HCS products are higher in calories and sugar than non-HCS products within the same product category. For example, Meiji Strawberry Milk (a popular brand in Singapore) bears the HCS and a tagline displaying 'lower in saturated fat'. Yet, it has $69 \mathrm{kcal}$ per $100 \mathrm{ml}$ compared to Pepsi's $42 \mathrm{kcal}$ per $100 \mathrm{ml}$. Assuming no offsetting behavior, drinking a Pepsi would be a better choice if the end goal were to reduce rates of obesity. So, even if the HCS improves diet quality, it may inadvertently increase caloric intake.

One strategy to address this potential unintended consequence is to supplement the HCS with FOP information on calorie content. There is prior evidence that showing calorie information in the form of the amount of activity time required to burn off the calories of a single serving of a product, termed physical activity equivalents (PAE), reduces total calories purchased $(3,4)$. Hence, a FOP label showing PAEs could address the potentially unintended negative consequences that may arise with the HCS.

To date, no published study has evaluated the effects of the HCS. Using a fully functional on-line grocery store, we test the effects of the HCS alone and in combination with a PAE FOP label on calories per serving purchased (primary outcome) and on other measures of diet quality (secondary outcomes). We hypothesize that calories per serving will be lowest in the HCS plus PAE labelling condition but that both labelling arms will positively influence diet quality relative to a no-label control condition. We further hypothesize that hunger and being unhappy at the time of the shop will moderate the effectiveness of the labels given that negative mood and hunger are associated with greater impulsivity (5). Thus, shoppers with these attributes may be more likely to ignore the labels altogether. 


\section{Methods}

\section{Study design and participants}

This was a crossover randomized controlled trial conducted via an online grocery store in Singapore. Participants were recruited online via Facebook advertisements from January to April 2019. Prospective participants were directed from recruitment advertisements to the study website (https://nusmart.dukenus.edu.sg/DIET) and asked to complete an online screener to determine their eligibility. Potential participants were eligible to participate if they were Singapore residents 21 years of age or above, and the primary grocery shopper for their household.

Potential participants who were both interested and eligible were then asked to complete: 1) a registration form containing name, delivery address, mobile number and email address; 2 ) an online consent form; and 3) the baseline questionnaire. Upon completion of the three forms, the website created the participant account and unique identification number for use throughout the study. Participants then received an automated email with their unique login details and were asked to logon to the NUSMart online grocery store to complete the first of three shopping tasks.

The study was conducted according to the guidelines laid down in the Declaration of Helsinki and all procedures were approved by the National University of Singapore Institutional Review Board Reference Code: S-18-073. Informed consent was obtained from all subjects. The trial was registered on the American Economic Association's registry for randomized controlled trials, RCT ID: AEARCTR- 0002883; Registered July 09, 2018. The protocol was not amended following commencement of the trial and is available online.

\section{NUSMart Online Grocery Store}

NUSMart is an online experimental grocery store developed by the study team and used to run the present trial (https://nusmart.duke-nus.edu.sg/DIET). At the time of the trial, NUSMart contained over 4,000 food and beverage products commonly purchased at local supermarkets in Singapore. The web store was designed to mirror actual web-based grocery stores in Singapore, such as FairPrice Online (https://fairprice.com.sg), in both look and feel. It contained products across 26 major food and beverage categories, further subset into 111 sub-categories for easier shopping. All products include pictures of the items, current retail price and product descriptions. NIP and product information are available on clickthrough. NUSMart operates similar to other online grocery stores in that participants fill a cart with products as they shop and have the ability to add and remove products, and review purchases before hitting the checkout button.

\section{Randomisation and masking}

Using a within-person crossover design, participants were randomly assigned to one of six intervention sequences, which included the order of the three shops and when the actual purchase would take place, via random permuted blocks of size three with equal allocation for the six sequences (see Additional File 
1 Table A1) by a computer program. Participants were blinded to intervention allocation, which was allocated via the NUSMart system. Allocation results were recorded within NUSMart and all investigators, including the data analyst, were blinded to group allocation.

\section{Procedures}

Arm 1 was the Control condition, which did not display FOP labels on any products. Arm 2 (termed HCSonly) displayed the HCS on eligible products, crossed referenced via the Health Promotion Board's HCS database (https://www.hpb.gov.sg/food-beverage/healthier-choice-symbol). Out of the 4,177 products available on NUSMart, 311 (7.45\%) carried the HCS. This was comprised of 150 foods and 161 beverages. Arm 3 displayed the HCS on eligible products as in Arm 2 and the PAE label on all products (termed HCS + PAE). PAE was calculated as the minutes required to burn off the calories of a single serving for a $73 \mathrm{~kg}$ person jogging at $8 \mathrm{~km}$ per hour.

For the study, we designed a simple PAE logo (Additional File 1 Figure A1) and added a description encoded into the NUSMart user interface to ensure that participants would understand the contents of the label. Participants saw the following description whenever their cursor hovered over the PAE label: "The Physical Activity Equivalent (PAE) refers to the number of minutes that a typical adult would need to jog to burn off the calories associated with one serving of the product." Previous studies have shown this labelling approach to be effective $(6,7)$.

The labels were displayed at the bottom of the product images. Figure 2 shows examples of what participants saw in each arm for the same product.

All participants were exposed once to each of the three shopping conditions (1xControl, 1 xHCS-only, $1 \mathrm{xHCS}+\mathrm{PAE}$ ) in random order. Participants were asked to shop once a week over a three-week period and were told on enrollment that they would need to purchase at least one and up to all three of their grocery orders. Following each shopping task, participants completed a brief survey to assess their mood and hunger level. 'Mood' took the values 1-5 where 1 was 'very happy' and 5 was 'very unhappy'. 'Hunger' took the values 1-10, where 1 was 'not at all hungry' and 10 was 'extremely hungry'. We used this information to determine if being either unhappy or hungry at the time of shopping moderated the effect of the labels. After completing the survey, participants spun a "Wheel of Purchase" to determine if they had to purchase their order. This was to ensure that there was a positive probability of having to purchase and receive the chosen products, thereby increasing the chance that product selections were an accurate reflection of participants' actual shopping behavior.

For each shop, there was a minimum and maximum spend requirement of SGD50 and SGD250 respectively. This was to ensure that participants completed a typical weekly grocery order and to make the study more manageable given that the orders, when necessary, were repurchased by the study team using an external online grocery store, RedMart (https://redmart.lazada.sg). Participants who completed all study elements were rewarded with SGD75 worth of electronic vouchers from an online marketplace.

\section{Outcomes}


The primary outcome is the average calories per serving purchased ( $\mathrm{kcal}$ per serving). We also assessed the following secondary outcomes:

- Proportion of HCS labelled products purchased (or would have been if not in control arm);

- Total Calories per shopping trip (kcal);

- Diet quality per shopping trip as measured by the Grocery Purchase Quality Index-2016 (GQPI-2016) and weighted average Nutri-Score;

- Sugar (g), Sodium (mg), and Saturated Fat (g) per serving;

- Calories per dollar (kcal per dollar) spent.

The Grocery Purchase Quality Index-2016 (GPQI-2016) contains 11 different food components with eight components scored based on adequacy and three moderation components. We followed the standard GPQI-2016 scoring methods by mapping NUSMart's subcategories to USDA food plan categories and then to the GPQI components (8). Each component was scored based on the deviation of the observed expenditure share of each component and the expected expenditure share, and the scores were totaled up to generate the final GPQI-2016 score for each participant's weekly grocery order.

We applied the standard Nutri-Score algorithm to assign a grade to each product (9-11). This algorithm assigns a score of $A$ to $E$ based on nutritional quality, which we recoded to 5 to 1 and then calculated an average score for each participant's weekly grocery order, weighted by the number of servings of each product.

Prior to conducting the analysis, we standardized the serving size by using the mean serving size within each subcategory. This standardization ensures that similar products are compared equally as serving sizes can be arbitrarily set by the manufacturers. Missing values for nutritional information were imputed using the median value of the non-missing nutrients within the product subcategory. The median was used to avoid the imputed value being susceptible to outliers, which is possible for some subcategories that contain a small number of products.

\section{Statistical Analysis}

\section{Sample Size}

The sample size was estimated based on an the ability to detect a standardized effect size of 0.3 in calories per serving between any two arms with $80 \%$ power, $5 \%$ (two-sided) level of significance, and a correlation of 0.5 in purchases across the three shops. Accounting for a $20 \%$ attrition rate based on prior studies using NUSMart (12), we estimated that the required sample size was 108 participants. Lower attrition would allow for a smaller sample size.

\section{Model}

To test our hypotheses, a first difference regression model was used with coefficients estimated via Ordinary Least Squares (OLS) with errors clustered at the individual level to account for correlation within 
individuals across shops. Therefore, each participant generates two observations, with each dependent variable being the difference in the outcome for each treatment condition (HCS or HCS + PAE) relative to the Control condition. This difference was calculated by subtracting the control arm outcome from the treatment arm outcome. We tested the effects of the labels by applying the following first-differenced model separately for each outcome of interest:

$\Delta$ outcome relative to control ${ }_{i t}=\alpha+\beta_{A} P A E_{i t}+\epsilon_{i t}$

which exploits the repeated observations of individuals by differencing out time invariant heterogeneity (e.g., age, health consciousness etc.) within individuals. The constant term a represents the incremental effect of the HCS condition relative to control. $P A E_{i t}$ is a dummy variable that is set to one when the difference in outcome is between the HCS + PAE condition and control condition. $\beta_{A}$ represents the incremental effect of the HCS + PAE condition relative to HCS only. $\varepsilon_{i t}$ is the error term for each individual, $\mathrm{i}$, and treatment condition, $\mathrm{t}$. To account for the potential impact of hunger and mood on the primary outcome, the following model was employed:

\section{$\Delta$ outcome relative to control ${ }_{i t}$}

$$
=\alpha+\beta_{A} P A E_{i t}+\beta_{B} \text { Moderator }_{i t}+\beta_{C} P A E_{i t} * \text { Moderator }_{i t}+\epsilon_{i t}
$$

where the additional Moderator $_{i t}$ term is a binary variable equal to one when the participants are not happy or hungry. We defined hungry and unhappy participants as those who had scores above the median. Separate analyses were run for each moderator. All analyses were run in Stata Version 15.2 (Stata Corp LP, College Station, TX).

\section{Results}

Participant recruitment and randomization is presented in Fig. 3. From January 2019 to April 2019, 117 participants began the shopping exercise and 12 participants (10\%) did not complete the three shops. Of the 12, 10 completed one shop only and their data could not be analyzed given the first difference design; two participants finished two shops but only one of them had a control shop and could thus be included; resulting in a total of 11 dropouts. This resulted in 317 unique shops from 106 participants, we could then generate 211 differences between treatment condition and control condition. Recruitment ended when sample size was reached. Table 1 presents the characteristics of the included sample. The sample was largely ethnic Chinese ( $90 \%$ ) with mean age of 36 years $(S D=6 \cdot 2)$. The average body mass index (BMI) was $23.7 \mathrm{~kg} / \mathrm{m}^{2}(\mathrm{SD}=4 \cdot 6)$ and the majority $(66 \%)$ were female. Participants were highly educated with $83 \%$ having at least a university degree. 


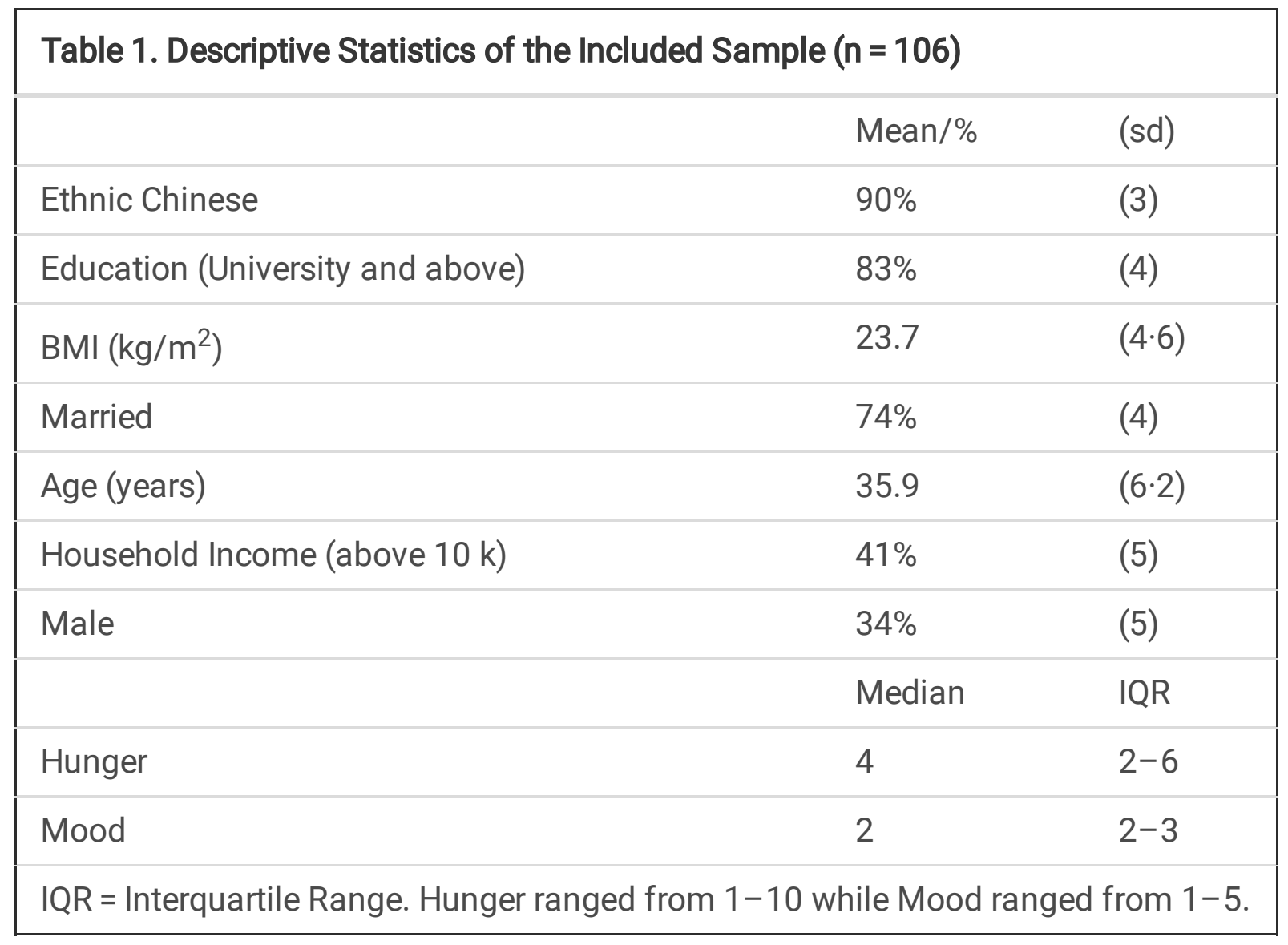

Table 2 presents the unadjusted values of the primary and secondary outcomes for the Control condition. Average calories per serving was $153 \mathrm{kcal}$ and the proportion of HCS products purchased was $14 \%$. Diet quality was 37.53 as measured by GPQI and 3.12 (slightly above a $\mathrm{C}$ grade) based on the weighted average Nutri-Score value of purchased products.

\section{Table 2. Unadjusted values of the primary and secondary dietary outcome variables per shop in the Control condition}

\begin{tabular}{|lll|}
\hline Measure & Mean & $95 \% \mathrm{Cl}$ \\
\hline Calories (kcal) per serving & 153 & $140-166$ \\
\hline Proportion of Heathier Choice Symbol products purchased & 0.14 & $0.11-0.17$ \\
\hline Total Calories (kcal) & 19,914 & $16760-23086$ \\
\hline Diet quality via Grocery Purchase Quality Index & 37.53 & $35.84-39.21$ \\
\hline Diet quality via avg. Nutri-Score & 3.12 & $2.96-3.27$ \\
\hline Sugar (g) per serving & 5.46 & $4.65-6.27$ \\
\hline Sodium (mg) per serving & 270 & $213-326$ \\
\hline Saturated Fats (g) per serving & 2.06 & $1.69-2.44$ \\
\hline Calories per dollar (kcal/SGD) & 378 & $318-437$ \\
\hline
\end{tabular}


Table 3 uses the regression results for the 211 observations to present the mean difference and 95\% confidence intervals comparing each arm to the other for the calorie outcomes, proportion of HCS products purchased and for measures of diet quality, with the regression results for the remaining secondary outcomes included in the Additional File 1 Table A2. From the results in columns 1 and 3 , we cannot reject the null hypothesis that there is no difference in calories per serving (primary) or total calories purchased across the three shopping conditions.

This is not to say the HCS did not influence purchasing behavior. Table 3 Column 2 reveals that the HCS increases the proportion of labelled products purchased by 5 percentage points ( $95 \% \mathrm{Cl} 1 \%$ : 9\%), from $14-19 \%$, relative to the control arm, without the inclusion of PAEs. However, this change in shopping behavior did not appear to significantly affect calories purchased, measures of diet quality, or nutrients in purchased foods (Table 3 and Additional File 1 Table A2). After adding the PAE logo, neither the proportion of labelled products purchased nor any of the other measures are statistically different from the control arm.

The moderator analyses (Additional File 1 Table A3) focusing on the primary outcome revealed no statistically significant differences by mood or hunger at the time of shopping, although this may be due to a lack of statistical power.

\section{Table 3. Estimated effects of HCS and HCS + PAE labels on characteristics of purchased foods and beverages.}

\begin{tabular}{|c|c|c|c|c|c|}
\hline & 1 & 2 & 3 & 4 & 5 \\
\hline & $\begin{array}{l}\text { Calories (kcal) per } \\
\text { serving }\end{array}$ & $\begin{array}{l}\text { HCS } \\
\text { proportion }\end{array}$ & $\begin{array}{l}\text { Total Calories } \\
\text { (kcal) }\end{array}$ & GPQI & $\begin{array}{l}\text { Average Nutri- } \\
\text { Score }\end{array}$ \\
\hline \multirow{2}{*}{$\begin{array}{l}\text { HCS + PAE vs } \\
\text { HCS }\end{array}$} & $4 \cdot 69$ & $-0 \cdot 04$ & 1,229 & $-0 \cdot 67$ & 0.05 \\
\hline & $(-10 \cdot 63: 20 \cdot 01)$ & $\begin{array}{l}(-0 \cdot 07: \\
0 \cdot 00)\end{array}$ & $\begin{array}{l}(-1,829: \\
4,287)\end{array}$ & $\begin{array}{l}(-2 \cdot 45: \\
1 \cdot 12)\end{array}$ & $(-0 \cdot 15: 0 \cdot 25)$ \\
\hline \multirow[t]{2}{*}{ HCS vs Control } & $3 \cdot 45$ & $0.05^{\star}$ & $-2,633$ & $0 \cdot 49$ & -0.01 \\
\hline & $(-12 \cdot 52: 19 \cdot 43)$ & $\begin{array}{l}(0.01: \\
0.09)\end{array}$ & $(-5,724: 458)$ & $\begin{array}{l}(-1 \cdot 21: \\
2 \cdot 19)\end{array}$ & $(-0 \cdot 21: 0 \cdot 19)$ \\
\hline \multirow{2}{*}{$\begin{array}{l}\text { HCS + PAE vs } \\
\text { Control }\end{array}$} & $8 \cdot 14$ & 0.02 & -1404 & $-0 \cdot 18$ & 0.04 \\
\hline & $(-5 \cdot 25: 21 \cdot 54)$ & $\begin{array}{l}(-0.02: \\
0.05)\end{array}$ & $(-5187: 2379)$ & $\begin{array}{l}(-1.94: \\
1.59)\end{array}$ & $(-0.14: 0.23)$ \\
\hline
\end{tabular}


$95 \% \mathrm{Cl}$ in parentheses. $* \star p<0 \cdot 01, * p<0 \cdot 05 . \mathrm{HCS}=$ Heathier Choice Symbol. PAE $=$ Physical Activity Equivalent. GPQI = Grocery Purchase Quality Index

It is worth restating that the lack of effects is not because the HCS did not influence food and beverage purchasing patterns. As noted above, the HCS without PAE increased the number of HCS products purchased by more than a third. However, a closer look at the products purchased reveals why this difference in purchasing patterns did not influence caloric intake or any measure of diet quality.

Focusing on beverages only, Table 4 compares the calories per serving and nutritional characteristics of the $84 \mathrm{HCS}$ beverages purchased during the experiment to that of the 153 non-HCS beverages purchased. In most cases, the confidence intervals overlapped. However, consistent with the strawberry milk example in the introduction, the HCS products purchased had greater amounts of sugar per serving and less saturated fat.

\begin{tabular}{|c|c|c|c|c|c|}
\hline Nutrient & $\mathrm{HCS}$ & Mean & $95 \% \mathrm{Cl}$ & Difference in mean & $\begin{array}{l}\text { p-value } \\
\text { (t-test) }\end{array}$ \\
\hline \multirow[t]{2}{*}{ Energy (kcal) } & $\mathrm{HCS}$ & 110 & $88-131$ & -10 & 0.45 \\
\hline & non-HCS & 99 & $84-115$ & & \\
\hline \multirow[t]{2}{*}{ Sugar (g) } & $\mathrm{HCS}$ & $15 \cdot 17$ & $13 \cdot 82-16 \cdot 52$ & $-2 \cdot 30$ & $\cdot 05^{\star}$ \\
\hline & non-HCS & $12 \cdot 87$ & $11 \cdot 04-14 \cdot 70$ & & \\
\hline \multirow[t]{2}{*}{ Saturated Fats (g) } & $\mathrm{HCS}$ & $0 \cdot 78$ & $0.57-1.00$ & 0.83 & $\cdot 01^{*}$ \\
\hline & non-HCS & $1 \cdot 61$ & $1 \cdot 07-2 \cdot 15$ & & \\
\hline \multirow[t]{2}{*}{ Sodium (mg) } & $\mathrm{HCS}$ & $61 \cdot 22$ & $51-72$ & 2 & $0 \cdot 83$ \\
\hline & non-HCS & 63.06 & $50-76$ & & \\
\hline \multirow[t]{2}{*}{ Protein (g) } & $\mathrm{HCS}$ & $3 \cdot 60$ & $2 \cdot 43-4 \cdot 78$ & $-1 \cdot 11$ & $0 \cdot 12$ \\
\hline & non-HCS & $2 \cdot 49$ & $1 \cdot 70-3 \cdot 27$ & & \\
\hline \multirow[t]{2}{*}{ Total Fats (g) } & $\mathrm{HCS}$ & $1 \cdot 76$ & $1 \cdot 11-2 \cdot 41$ & 0.69 & $0 \cdot 22$ \\
\hline & non-HCS & $2 \cdot 45$ & $1 \cdot 56-3.33$ & & \\
\hline
\end{tabular}

\section{Discussion}

This study shows that the HCS front-of-pack label does influence consumers' purchasing patterns towards buying a higher proportion of HCS products, but also shows that, as applied, we could not reject 
the hypothesis that it is not having the intended effect of reducing caloric intake, improving overall diet quality or positively influencing the consumption of any single nutrient. Adding the PAE label does not change this conclusion and appears to dilute the effect of the HCS in influencing purchases of labelled products.

In the introduction we proposed several possible reasons for the potential lack of effectiveness of the HCS. These remain plausible but it appears an additional explanation for the lack of effectiveness is that the HCS products purchased may be, on average, no healthier than the non-HCS products purchased. The results because HCS products that are healthier in some dimensions are less healthy in others, as was shown in the strawberry milk example. Had the HCS focused on calories only or a single nutritional characteristic, it likely would have had the intended effect.

The Singapore government is aware that the HCS has not been effective in stemming rising rates of obesity and diabetes, and has recently proposed changes to the current labelling policy, including additional FOP nutrition labels on beverages (13). The new policy states that a new label will be added that will be colour coded with a summary grade based on the nutritional quality, with sugar being the main determinant. It will be mandatory for the worst offending beverages and optional for healthier drinks. This approach is similar to France's Nutri-Score (Fig. 4) label, and SSBs with D or E grade will be required to post the label. This would essentially operate like a warning label indicating that these are products to avoid.

By focusing primarily on sugar, the new label will tie more closely to obesity/diabetes than the current HCS, and thus should be more effective in reducing sugar intake. Moreover, focusing on the worse offending beverages does not induce the type of potentially offsetting behaviour that comes with a positive label such as the HCS. It also provides a clear signal to manufactures of what and how to reformulate to get a better rating.

These changes are promising but may be more effective if expanded to include both foods and beverages since there are plenty of discretionary foods that are very high in calories and sugar and with little nutritional value. It may also be necessary to remove the HCS as our results suggest that having two labels may confuse consumers and dilute the effects of each.

Our study has many strengths, including taking advantage of a fully functional web-based grocery store with a subset of purchases actually delivered to participants home, but also significant limitations. This includes being limited to a few shops and a non-representative sample of consumers. Future efforts should attempt to replicate these results using in-store purchases, such as from scanner data or household panels.

In summary, this study suggests that the HCS appears to influence food purchasing patterns but does not, either alone or in combination with a PAE label, appear to reduce caloric intake or improve overall diet quality. These findings suggest that the HCS, as currently applied, may be the wrong label for addressing rising rates of obesity and NCDs in Singapore. 


\section{Conclusion}

Our results suggest that the HCS influences purchasing patterns, but does not, either alone or in combination with a PAE label, appear to reduce caloric intake or improve overall diet quality. Our study suggests that the HCS may be ineffective in addressing rising rates of obesity and NCDs in Singapore. Findings are timely given that the Singapore government is mandating new measures to reduce sugar intake from sugar-sweetened beverages, including new FOP labels. Evidence from this study should be taken into consideration before any new labels are introduced.

\section{Abbreviations}

FOP:Front-of-pack; HCS:Healthier Choice Symbol; PAE:Physical Activity Equivalent; NIP:Nutrition Information Panel; GPQI-2016:Grocery Purchase Quality Index-2016; OLS:Ordinary Least Squares

\section{Declarations}

\section{Ethics approval and consent to participate}

This study was conducted according to the guidelines laid down in the Declaration of Helsinki and all procedures involving human subjects/patients were approved by the National University of Singapore Institutional Review Board Reference Code: S-18-073. Written informed consent was obtained from all subjects.

\section{Consent for publication}

Not applicable.

\section{Availability of data and materials}

De-identified participant data and any other datasets used and/or analyzed during the current study are available from the corresponding author on reasonable request.

\section{Competing interests}

The authors declare that they have no competing interests.

\section{Funding}

This work was funded by the National Medical Research Council (grant number NMRC-HSRG-2016-0060). The National Medical Research Council had no role in the study design, data collection, analysis and 
interpretation of the data, or the writing of the report. The research was conducted independently of the funders.

\section{Authors' contributions}

EAF conceived and designed the study. FJLA led data collection. WHMW and BD led data analysis. FJLA and WHMW wrote the first draft of the manuscript with input from BD. EAF, BD and RMVD revised the manuscript critically for important intellectual content. All authors read, provided input to, and approved the final version of the manuscript.

\section{Acknowledgements}

Not applicable.

\section{References}

1. Foo LL, Vijaya K, Sloan RA, Ling A. Obesity prevention and management: Singapore's experience. Obes Rev. 2013;14 Suppl 2:106-13. 2. Talati Z, Pettigrew S, Dixon H, Neal B, Ball K, Hughes C. Do health claims and front-of-pack labels lead to a positivity bias in unhealthy foods? Nutrients. 2016;8(12):787. 3. Bleich SN, Barry CL, Gary-Webb TL, Herring BJ. Reducing Sugar-Sweetened Beverage Consumption by Providing Caloric Information: How Black Adolescents Alter Their Purchases and Whether the Effects Persist. American journal of public health. 2014;104(12):2417-24. 4. James A, Adams-Huet B, Shah M. Menu Labels Displaying the Kilocalorie Content or the Exercise Equivalent: Effects on Energy Ordered and Consumed in Young Adults. American Journal of Health Promotion. 2015;29(5):294-302. 5. Atalay AS, Meloy MG. Retail therapy: A strategic effort to improve mood. Psychology and Marketing. 2011;28(6):638-59. 6. Hartley IE, Keast RSJ, Liem DG. Physical activity-equivalent label reduces consumption of discretionary snack foods. Public Health Nutr. 2018;21(8):1435-43. 7. Antonelli R, Viera AJ. Potential Effect of Physical Activity Calorie Equivalent (PACE) Labeling on Adult Fast Food Ordering and Exercise. PLoS One. 2015;10(7):e0134289. 8. Brewster PJ, Guenther PM, Jordan KC, Hurdle JF. The Grocery Purchase Quality Index-2016: An innovative approach to assessing grocery food purchases. Journal of Food Composition and Analysis. 2017;64:119-26. 9. Julià CR, Hercberg S. Development of a new front-of-pack nutrition label in France : the five-colour Nutri-Score. Catalina Roig Julià, Serge Hercberg. 2017;3(4). 10. France PH. Nutri-Score France2016 [updated 30 April 2019. Available from: http://www.santepubliquefrance.fr/Sante-publique-France/Nutri-Score. 11. Julia C, Etilé F, Hercberg S. Front-of-pack Nutri-Score labelling in France: an evidence-based policy. The Lancet Public Health. 2018;3(4). 12. Finkelstein E, Ang F, Doble B, Wong W, Dam R. A Randomized Controlled Trial Evaluating the Relative Effectiveness of the Multiple Traffic Light and Nutri-Score Front of Package Nutrition Labels. Nutrients. 2019;11:2236. 13. $\mathrm{MOH}$ to introduce measures to reduce sugar intake from pre-packaged sugar-sweetened beverages [press release]. Singapore: Ministry of Health, 10 October 20192019. 
Figures
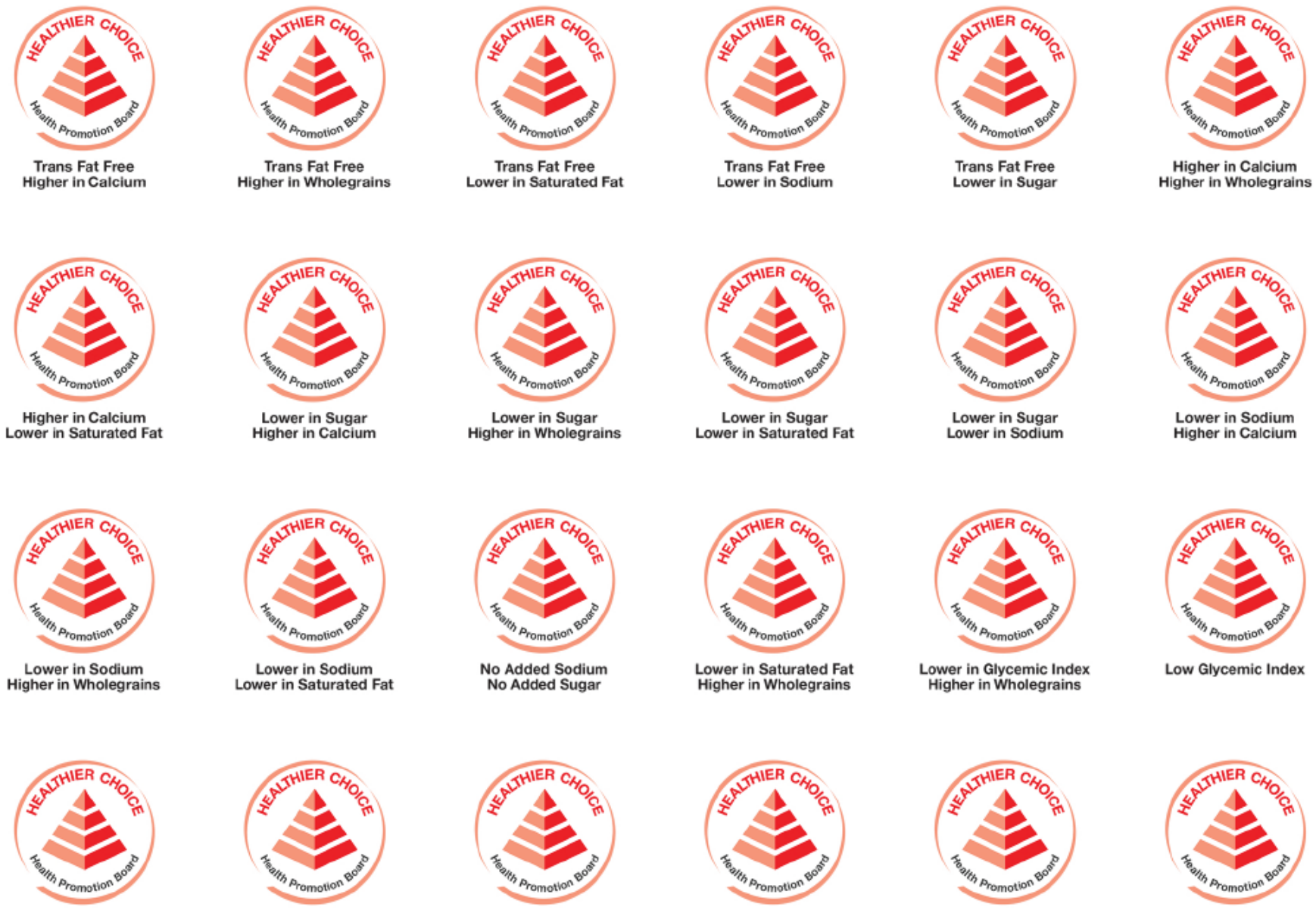

No Added Sodium

Lower in Sugar

Lower in Sodium

Lower in Saturated Fat

Higher in Calcium
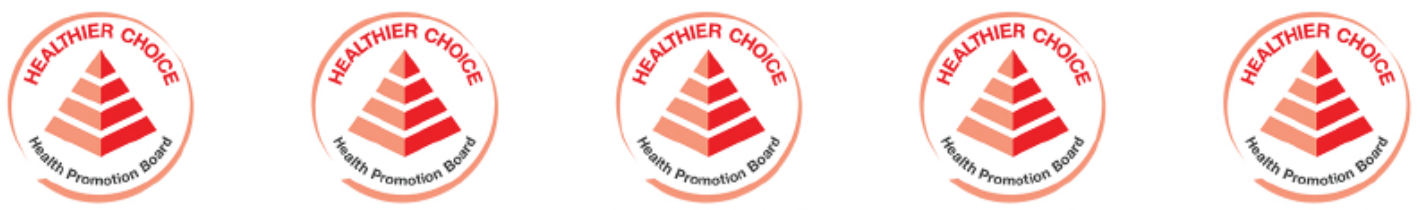

Less Than 100 Calories

Higher in Wholegrains

Lower in Cholesterol

Less Than 200 Calories

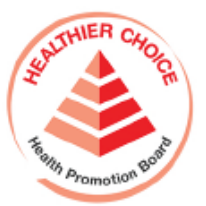

Eat $2+2$ servings of fruits and vegetables daily

\section{Figure 1}

30 Healthier Choice Symbol Variants 


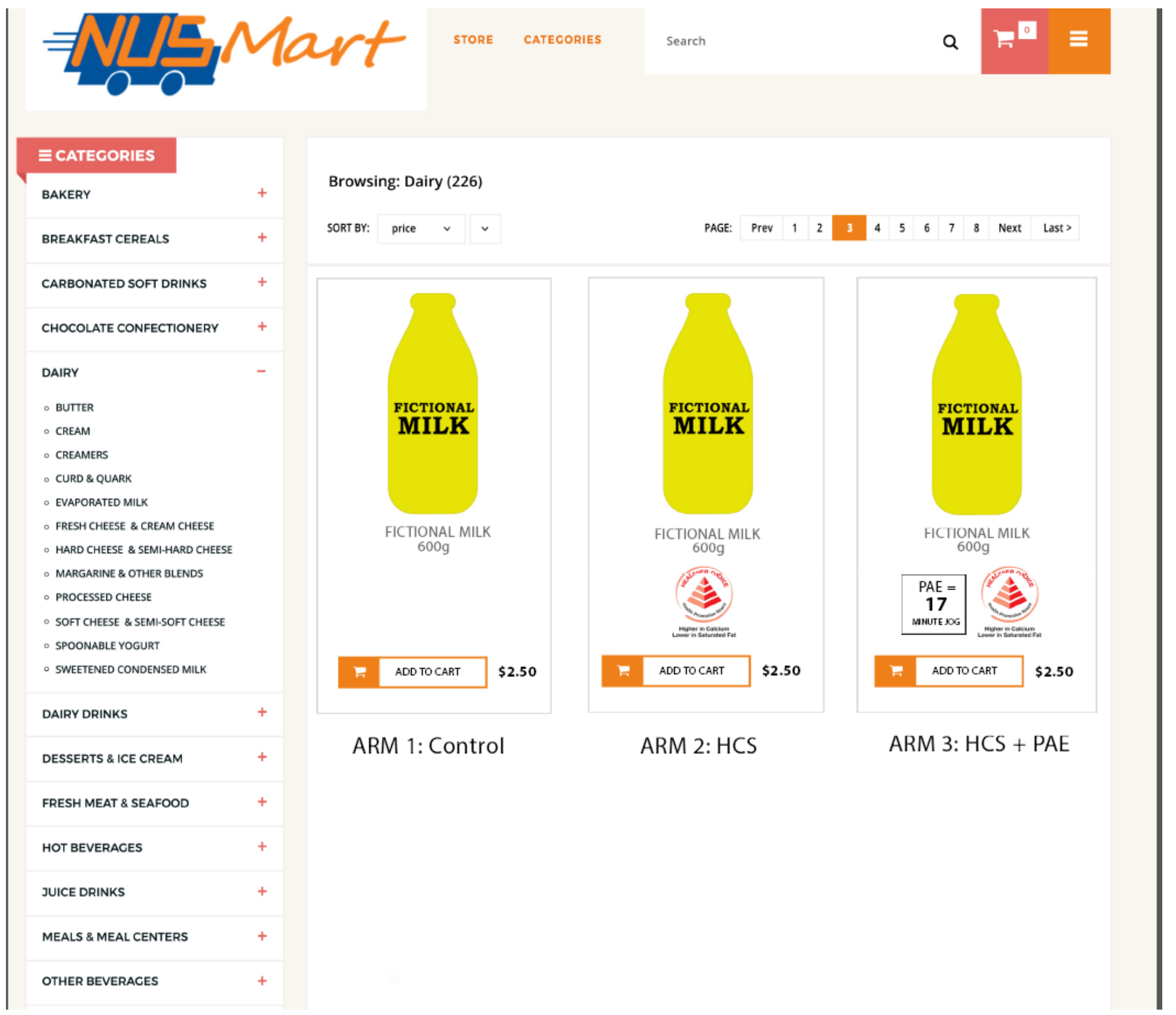

Figure 2

Example product from NUSMart showing how the labels were presented across the three study arms. 


\section{CONSORT 2010 Flow Diagram}

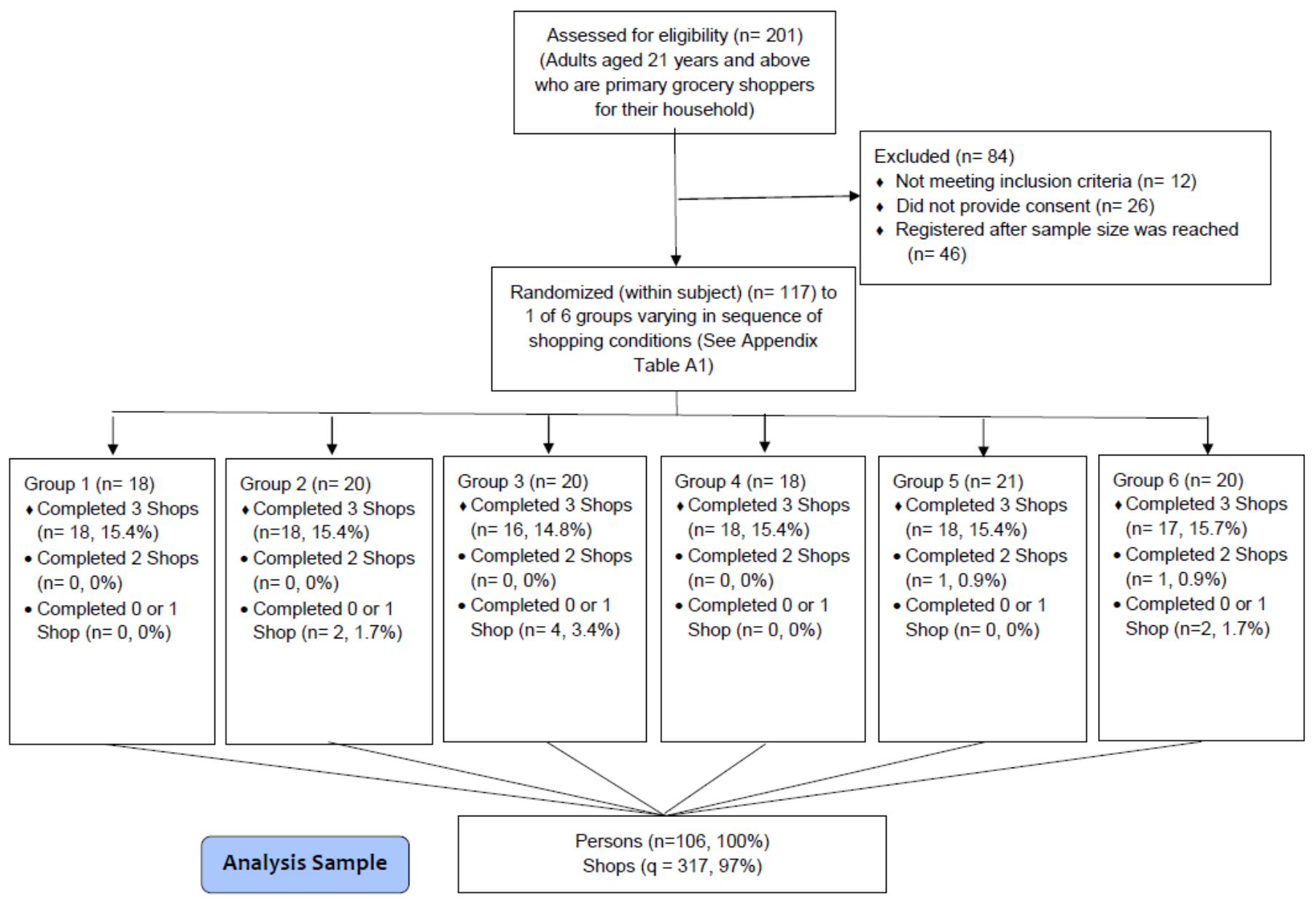

Figure 3

CONSORT Flow Diagram for participant recruitment and randomization. 


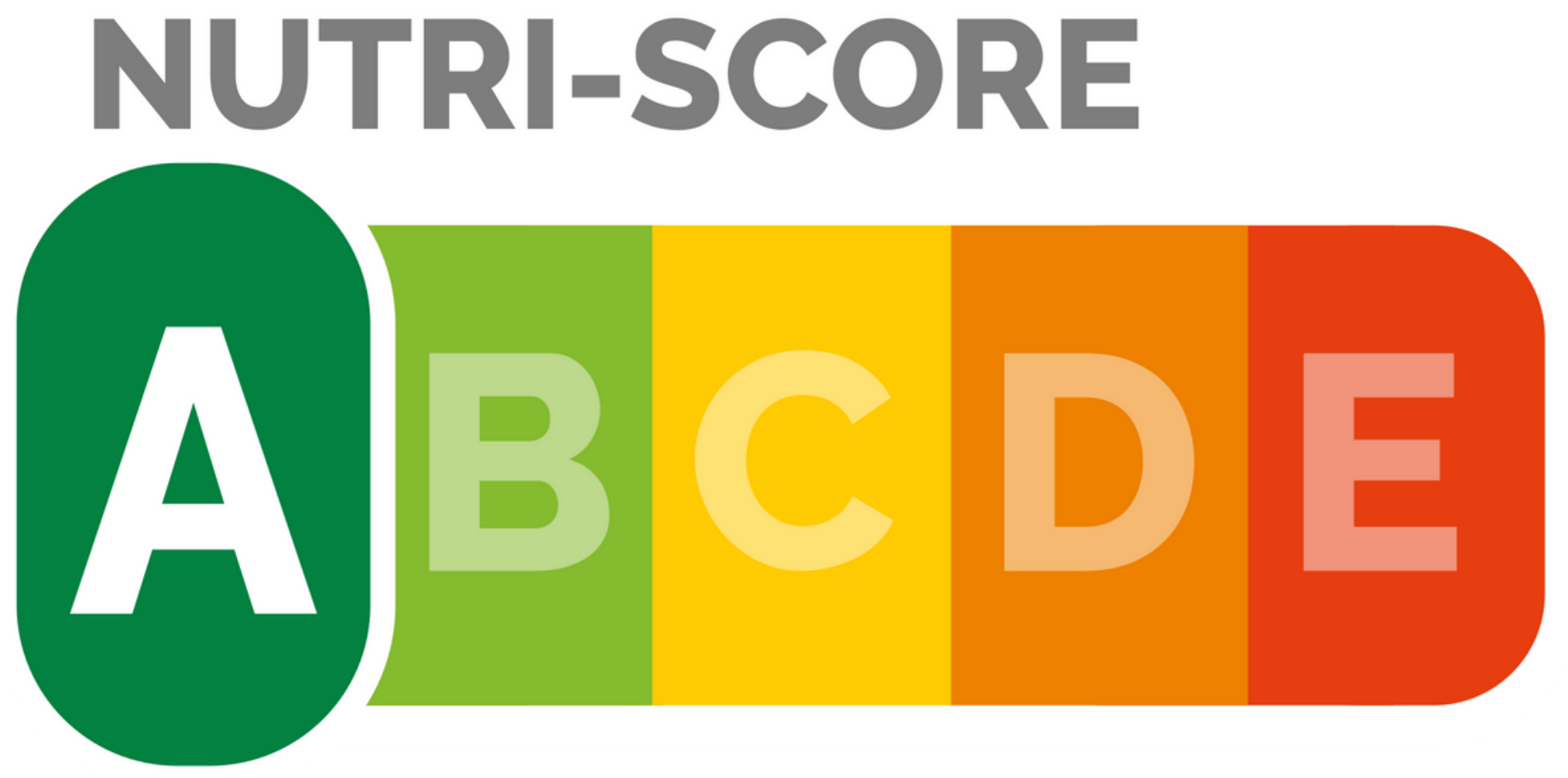

Figure 4

French Nutri-Score Label by Santé Publique France

\section{Supplementary Files}

This is a list of supplementary files associated with this preprint. Click to download.

- Additionalfile4TIDieRChecklistWord.docx

- AdditionalFile1Appendix.docx

- AdditionalFile3CONSORT2010Checklist.doc

- AdditionalFile2Sample.docx 